Volume and Issues Obtainable at Center for Sustainability Research and
Consultancy

\title{
Developmental Change of Approximate Number System Acuity (Keenness) Reveals Delay
}

Tayyaba Abid, Department of Behavioral Sciences $S^{3} \mathrm{H}$, National University of Sciences and Technology Islamabad, Pakistan and ABA Instructor (CLM) Applied and Behavioral Training Institute, UAE

*Saeeda Khanum, Department of Behavioral Sciences, $\mathrm{S}^{3} \mathrm{H}$, National University of Sciences and Technology Islamabad, Pakistan

*Corresponding author's email: saeeda.khanum@s3h.nust.edu.pk

\begin{tabular}{l}
\hline ARTICLE DETAILS \\
\hline History \\
Revised format: May 2021 \\
Available Online: Jun 2021
\end{tabular}

\section{Keywords}

Approximate Number

System, Acuity, Keenness,

Development, Change

\section{JEL Classification}

I20,I25

\begin{abstract}
Purpose: Major aim of the study was to investigate the development of mathematical thinking and processing in Pakistani sample. Particular focus of the study was to figure out whether Pakistani people across various age groups process numbers with similar sophistication as their western counter parts from developed countries. Mathematics plays huge role in the development of the society and research evidence in this context from Pakistan is scarce.
\end{abstract}

Design/Methodology/Approach: 261 participants ranging from 5 to 72 years of age participated in the study. Panamath task being the robust measure of ANS acuity was administered.

Findings: Results revealed that numerical acuity got precise with an increase in age. However, most sophisticated acuity has been shown around age 46-50 as compared to the western population showing its peak around 30 years of age. Delay in developing most sophisticated approximate number system acuity across the groups as compared to the trend reported in the western population raises many questions in terms of cultural variations and practices contributing to the development of number sense.

Implications/Originality/Value: We need to improve our mathematical learning and teaching practices so that it could be helpful in economic growth in turn by better mathematical performance across various age groups. On a general note, economical practices, math related curriculum policies, lack in math related games, math practices at home and at educational institutions with varying level of curriculum and pedagogical practices might be a contributor for this trend. The study has important implications for understanding the development of number sense cross-culturally keeping in view the evidence from various cultures.

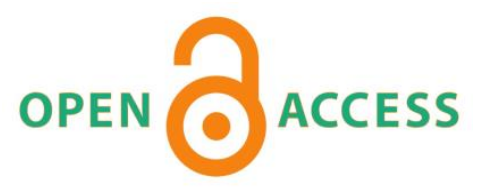

(C) 2021 The authors, under a Creative Commons AttributionNonCommercial 4.0

Recommended citation: Abid, T. and Khanum, S. (2021). Developmental Change Of Approximate Number System Acuity (Keenness) Reveals Delay. Journal of Business and Social Review in Emerging Economies, 7 (2), 359-368. 


\section{Introduction}

Approximate number system (ANS) keenness also called acuity is related to children's mathematics achievement (Halberda, Mazzocco, \& Feigenson, 2008). This system plays a foundational role in the development of numerical ability and school math performance. ANS is present early in development and newborn babies have been reported to process numbers approximately (Hyde \& Spelke, 2011; Izard, Sann, Spelke \& Steri, 2009; Libertus \& Brannon, 2009; 2010; Lipton \& Spelke, 2003; Xu \& Spelke, 2000). It is functional before formal schooling (Gilmore, McCarthy, \& Spelke, 2007) and continues to develop throughout the development. The increase in ANS acuity (keenness) continues throughout childhood (Halberda \& Feigenson, 2008; Piazza et al., 2010), until around 30 years of age (Halberda, Ly, Wilmer, Naiman, \& Germine, 2012).

The approximate number system operates independently of education and language for processing number (Gordon, 2004; Pica, Lemer, \& Izard, \& Dehaene, 2004). Correlational research evidence has revealed that approximate number system is correlated with later math achievement (Bonny \& Loureneo, 2013; Chen \& Li, 2014; Fazio, Bailey, Thompson, \& Siegler, 2014; Feigenson, Libertus, \& Halberda, 2013; Fuhs \& McNeil, 2013; Halberda, et al., 2008; Libertus, Feigenson, \& Halberda, 2013b; Libertus, Odic, \& Halberda, 2012; Inglis, Attridge, Batchelor, \& Gilmore, 2011; Libertus, Feigenson, \& Halberda, 2011; Mazzocco, Feigenson, \& Halberda, 2011a).

Approximate number system is predictor of later math achievement (Agrillo, Piffer \& Adriano, 2013; Libertus, Feigenson, \& Halberda, 2013a; Mazzocco, Feigenson, \& Halberda, 2011b, Sasanguie, Van den Bussche, \& Reynvoet, 2012; Starr, Libertus, \& Brannon, 2013).

However, other studies have not found approximate number system performance to be correlated or predictor of later math (De Smedt, Noel, Gilmore, \& Ansari, 2013; De Smedt \& Gilmore, 2011; Gilmore, et al., 2013; Holloway \& Ansari, 2009; Landerl \& Kolle, 2009; Lyons, Ansari, \& Beilock, 2012; Rouselle \& No“el, 2007; Solt'esz, Szucs \& Szucs, 2010; Sasanguie, Gobel, Moll, Smets, \& Reynvoet, 2013; Vanbinst, Ghesquiere, \& De Smedt, 2012).

Mixed results have been reported by other researchers (Sasanguie, De Smedt, Defever, \& Reynvoet, 2012; Gilmore, Attridge, \& Inglis, 2011). The non-symbolic numerical magnitude was predictively related to arithmetic proficiency in second grade but not in first grade (Desoete, Ceulemans, Weerdt, \& Pieters, 2010; Vanbinst, Ghesquiere, \& De Smedt, 2015). Children map on approximate number system when they have to solve symbolic math problems (Brankaer, Ghesquiere, \& Smedt, 2014a; Castronovo, \& Gobel, 2012; Mundy \& Gilmore, \& Spelke, 2009).

The meta-analysis (Schneider, et al., 2017; Chen, \& Li, 2014; Fazio, et al., 2014) and review research evidence has also reported a positive correlation between approximate number system acuity and math performance (De Smedt, Noel, Gilmore, \& Ansari, 2013). Individuals with math learning difficulties have been reported to have impaired approximate number sense (Brankaer, Ghesquiere, \& Smedt, 2014b; Mazzocco, et al., 2011a). Research evidence indicates that approximate number system training can play important role in math improvement. Approximate number system training has been reported to enhance children's performance on symbolic math (Dillon, Kannan, Dean, Spelke, \& Duflo, 2017; Hyde, Khanum, \& Spelke, 2014; Park, \& Brannon, 2013; 2014). Education has also been reported to alternatively enhance the approximate number system (Piazza, Pica, Izard, Spelke, \& Dehaene, 2013).

\section{Purpose of the Present Study}

The developmental trajectory of the approximate number system has been investigated in western culture (Halberda \& Feigenson, 2008; Halberda, et al., 2012). However, no such research evidence has been informed from the Pakistani context specifically indicating the 
developmental trajectory of approximate number system acuity except a comparative study of approximate number system acuity first-grade children from USA and Pakistan (Khanum \& Hanif, 2014). Research evidence from the USA (Hyde, et al., 2014) and Pakistani (Khanum, Hanif, Berteletti, Spelke, \& Hyde, 2016) first-grade children showed that Pakistani children had slightly lower Weber fraction as compared to USA children although both were trained under similar training conditions (Table1). These results led to an intriguing question about numerical acuity development in various cultures subject to differences in many aspects.

Table 1

Comparison of approximate number system acuity from Hyde, et al., 2014 and Khanum et al., 2016

\begin{tabular}{|l|l|l|}
\hline & $\begin{array}{l}\text { Hyde, et al., 2014 } \\
\text { (American) }\end{array}$ & $\begin{array}{l}\text { Khanum et al., 2016 } \\
\text { (Pakistan) }\end{array}$ \\
\hline Experimental Conditions & Weber Fraction & Weber Fraction \\
\hline Non-symbolic approximate addition & .17 & .19 \\
\hline Brightness Comparison & .17 & .20 \\
\hline Line Length addition & .21 & .23 \\
\hline
\end{tabular}

Above mentioned results raised the question whether Pakistani people will have lower approximate number system acuity throughout their life span as compared to the western sample (e.g., Halberda \& Feigenson, 2008; Halberda et al., 2012)? So, the current study has been carried out to investigate the developmental trajectory of the approximate number system acuity in Pakistan. Findings of research can have important implications for researchers indigenously as well as internationally.

\section{Method}

\section{Participants}

The total number of participants recruited for the study were 265 (133 males and 132 females' participants). Responses of seven participants were excluded from the main data due to child eye-related issues (1), participant withdrawal from the experiment (2), laptop/system failure (2), and researcher assistance to the respondents (2).

Data was collected from participants of varying backgrounds including, Airline ticket agents (2), Army officers (4), Teachers (14), Maids/ help at home (3), Software and IT engineers (39), Marketing and Finance experts (30), Doctors (14), Property dealers (4), Interior designer (1), Clerical staff (2), Drivers (7), Housewives (47), Lawyer (2), Security guards (3), Photographer (1), Laborer (5), Chef (1), students from community schools (8), private school (52), university (20), telecom engineers (2), and Librarian (2).

\section{Materials}

\section{Apparatus, Distance of Participant from}

The laptop was approximately $13 \mathrm{~cm}$. The participants were seated right in front of the laptop and they were allowed to adjust the screen angle and distance. The keyboard was covered with a black sheet except for the spacebar, F, and J keys. F was covered with yellow paper and $\mathrm{J}$ with blue paper. The space bar was covered with a white sheet.

\section{Panamath Task}

Approximate number sense acuity or keenness was measured through the Panamath task (Halberda et al., 2008). The beta version of the game was installed on the laptop having a screen size of 11.6 inches. The task involved sets of yellow and blue dots and the participants had to indicate through a button whether there were more yellow or blue dots. The mask 
display time for each trial was $200 \mathrm{~ms}$. Participants completed a varied total number of trials for participants varying with age groups as mentioned in table 1 . Weber fractions were calculated through a built-in macro (Halberda et al., 2008). The participants played the game with its default settings and medium difficulty level and audio feedback through beep (positive and negative) was given. However, instructions were given both verbally and on screen. The number of dots ranged between 5-21 dots. For kids, age range 5-7, the array of dots accompanied Sesame street characters. Dot set 1 (yellow dots) was accompanied by a big bird and dot set 2 (blue dots) by Grover. For age 8 and above, the arrays of dots appeared on the screen without any characters or borders. The ending screen presented the performance stats having Weber Fraction, Reaction Time, and graphical display of the results. The same apparatus was used for all the 265 participants. Further details are given at http://panamath.org.

\section{Procedure}

Participants were introduced to the experiment in a manner that they will be playing a computer gamer through which their number acuity can be measured. Instructions were given bilingually, in national language Urdu and English and they were explained about how to play the game.

The purpose of the experiment was explained to all participants in their native language Urdu. Informed consent was obtained from all participants. However, before presenting the consent form, permission was taken from each participant verbally. In the case of participants under 16 years of age; permission was taken from school higher authorities and parents as well.

Mode of instructions and experimental conditions were kept similar. All the queries were answered to avoid any confusion. Data from participants was collected in varied settings. However, the researcher ensured that the experimental conditions should remain similar during the task across participants. Each participant had the chance to play the game only once. Depending upon the age groups compensation was given to all participants.

\section{Analysis}

Data was analyzed after grouping the participants with an interval of around five years of age resulting in 13 age groups. The numerical acuity of the participants was analyzed for each group and it was analyzed across age groups (Table 2). Data analysis was carried out in terms of Weber fraction across various age groups. One-way ANOVA was carried out (Table 3) between the Weber fraction and Age.

Table 2

Age range, frequency, mean Weber fraction $\left(W^{* 1}\right)$, average reaction time $\left(\mathrm{RT}^{* 2}\right)$, and percent correct responses of the respondents $(\mathbf{n}=\mathbf{2 5 8})$

\begin{tabular}{|l|l|l|l|l|}
\hline Age Range & Frequency & Mean W & Mean RT & Percent Correct \\
\hline $5-10$ & 32 & .32 & 1494.56 & 12.4 \\
\hline $11-15$ & 23 & .25 & 939.71 & 8.9 \\
\hline $16-20$ & 14 & .19 & 779.62 & 5.4 \\
$21-25$ & 28 & .25 & 635.99 & 10.9 \\
$26-30$ & 18 & .22 & 710.63 & 7.0 \\
$31-35$ & 19 & .18 & 672.10 & 7.4 \\
$36-40$ & 22 & .19 & 825.35 & 8.5 \\
$41-45$ & 19 & .13 & 688.78 & 7.4 \\
$46-50$ & 16 & .16 & 625.72 & 6.2 \\
$51-55$ & 22 & .17 & 77.39 & 8.5 \\
$56-60$ & 18 & .16 & 942.99 & 7.0 \\
$61-65$ & 20 & .14 & 809.81 & 7.8 \\
$66+$ & 07 & & & 2.7 \\
& & & & \\
\hline
\end{tabular}




\begin{tabular}{|l|l|l|l|l|}
\hline Total & 258 & .21 & 847.00 & 100.0 \\
\hline
\end{tabular}

*1-Weber fraction indicated the keenness score of participants.

*2-Reaction time indicates the time participants took to respond to each trial

Table 3

One Way Analysis of Variance of Weber fraction* by age $(n=258)$

\begin{tabular}{|l|l|l|l|l|l|}
\hline & Sum of Squares & df & Mean Square & $\boldsymbol{F}$ & $\boldsymbol{p}$ \\
\hline Between Groups & .79 & 12 & .06 & 3.49 & .00 \\
\hline Within Groups & 4.63 & 245 & .01 & & \\
\hline Total & 5.43 & 257 & & & \\
\hline
\end{tabular}

*Weber fraction indicated the keenness score of participants, the lower values indicate the more sophisticated ability of number and the higher values indicated the less sophisticated ability of number

Results revealed that there was a significant difference between age groups in terms of Weber Fraction $\left(F(12,245)=3.49, p=.00, \eta_{p}{ }^{2}=.146\right)$. It showed that participant's precision of the approximate number system was increasing with age (Figure 1). Weber fraction values were getting more sophisticated (smaller decimal points indicate better performance) with increasing age. Post-hoc analysis revealed that the major significant difference in mean Weber fraction occurred around age 30 and above. Beyond age 30 Weber fractions was getting further sophisticated (Table 3). These results should be interpreted with caution keeping in view educated and non-educated individuals in the sample.

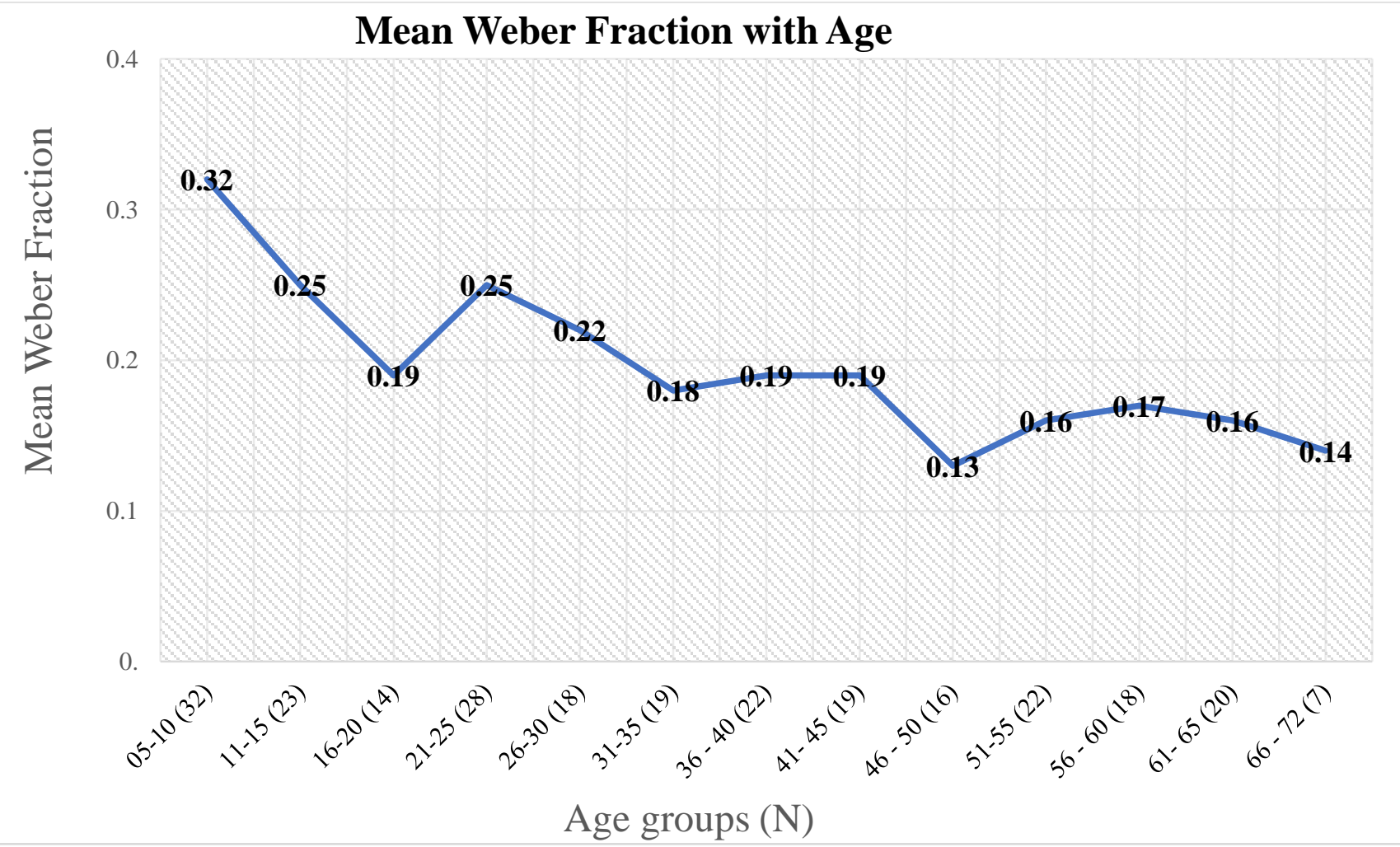

Figure 1. Weber Fraction of participants across age groups

Results revealed that that there was significant difference between age groups in terms of reaction time $\left(F(12,245)=11.18, p=.00, \eta_{p}{ }^{2}=.354\right)$. It showed that participant's reaction time on the Panamath task was decreasing with age although variably (Figure 2). Posthoc analysis revealed that reaction time performance was significantly different across all age groups. However, the mean reaction time (see Figure 2) of age group 46-50 was fastest. 
Table 4

One Way Analysis of Variance of average reaction time by age $(\mathbf{n}=\mathbf{2 5 8})$

\begin{tabular}{|l|l|l|l|l|l|}
\hline & Sum of Squares & df & Mean Square & $F$ & $P$ \\
\hline Between Groups & 17967649.67 & 12 & 1497304.13 & 11.18 & .00 \\
\hline Within Groups & 32790706.22 & 245 & 133839.61 & & \\
\hline Total & 50758355.90 & 257 & & & \\
\hline
\end{tabular}

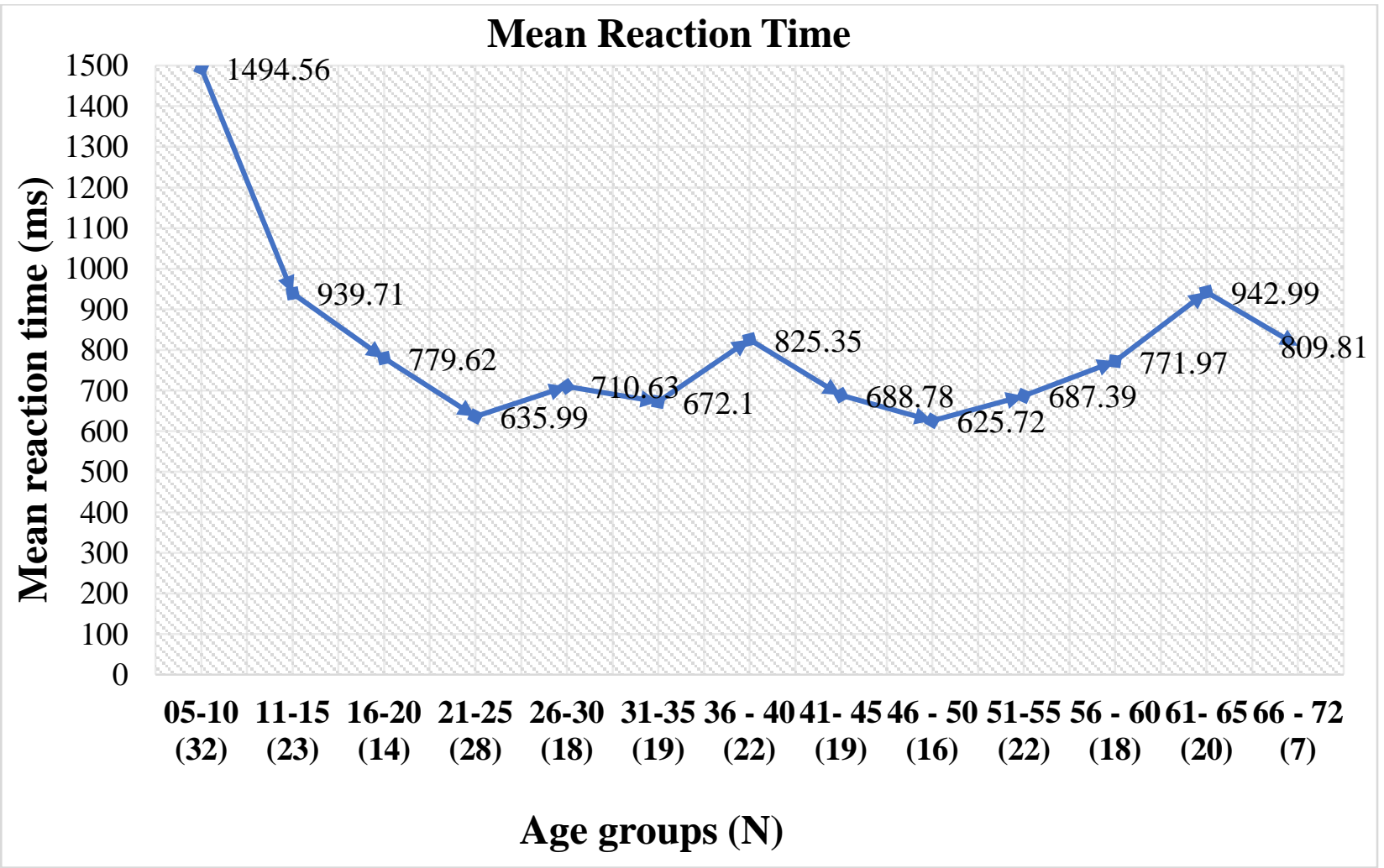

Figure 2: Mean reaction time accros age groups

\section{Conclusion and Discussion}

Results revealed that approximate number system acuity across various age groups got more precise with increasing age. These results are in line with the finding from other researchers who investigated the developmental trajectory of the approximate number system in western culture (Halberda \& Feigeonson, 2008; Halberda, et al., 2012; Odic, Libertus, Feigenson \& Halberda, 2013).

However, results revealed delayed acuity as compared to the western population and the most precise Weber fraction in the Pakistani sample has been shown around age group 46-50 ( $W=$ .13). Whereas in the USA, Weber fraction of adults group ranging from $18-32$ years of age has been reported as .11 (Halberda \& Feigenson, 2008). Similarly, optimal precision in the Weber fraction is around age 30 in Halberda et al., 2012.

The findings raise intriguing questions about the development of the approximate number system acuity in varying cultures and backgrounds. It is important to investigate why the Pakistani sample has shown a lower Weber fraction than the USA sample and why the most precise $\mathrm{W}$ has appeared so much delayed along the trajectory as compared to the USA sample. This trend of data indicates that although numerical acuity gets precise with age and many factors might be contributing to the precision along with age. Participants belonging to various backgrounds and educational levels can also be a contributing factor showing these trends of data other than developed and developing country context-related differences. 
These differences might also be attributed to economic factors, less technology exposure, and educational systems difference as compared to the USA. Home numeracy environment, mathrelated games, the importance of performance and achievement in math as compared to conceptual understanding of math, etc. can also be the contributing factors. This research evidence can have an important contribution in terms of figuring out the possible factors affecting approximate number system acuity as well as various strategies to improve this acuity either through training or education. Approximate number system acuity plays an important role in quantitative reasoning throughout the human life span. It has important implications for later mathematical achievement. Future research focusing on possible predictors of better numerical acuity and the cross-cultural aspect of the development of the approximate number system can shed further light on the developmental trajectory of the approximate number system.

\section{Acknowledgment}

The corresponding author conceptualized the idea, designed the research project. The second author collected data. Both authors analyzed and wrote the paper collaboratively. The authors declare that there is no conflict of interest.

\section{References}

Agrillo, C., Piffer, L., \& Adriano, A. (2013). Individual differences in non-symbolic numerical abilities predict mathematical achievements but contradict ATOM. Behavioral and Brain Functions, 9 (26).

Bonny, J.W., \& Loureneo, S.F. (2013). The approximate number system and its relation to early math achievement: evidence from the preschool years. Journal of Experimental Child Psychology, 114, 375-388.

Brankaer C., Ghesquière P., De Smedt, B. (2014b). Numerical magnitude processing deficits in children with mathematical difficulties are independent of intelligence. Research in Developmental Disabilities, 35(11), 2603-2613.

Brankaer, C., Ghesquiere, P., \& Smedt, B.D. (2014a). Children's mapping between nonsymbolic and symbolic numerical magnitudes and its association with timed and untimed tests of mathematics achievement. PLOS ONE, 9 (4), e93565.

Castronovo, J., \& Gobel, S.M. (2012). Impact of high mathematics education on the number sense. PLoS ONE, 7 (4), e33832.

Chen, Q., \& Li, J. (2014). Association between individual differences in non-symbolic number acuity and math performance: a meta-analysis. Acta Psychologica, 148, 163172.

De Smedt, B., \& Gilmore, C. K. (2011). Defective number module or impaired access? Numerical magnitude processing in first graders with mathematical difficulties. Journal of Experimental Child Psychology, 108, 278 - 292.

De Smedt, B., Noel, M.-P., Gilmore, C., \& Ansari, D. (2013). How do symbolic and nonsymbolic numerical magnitude processing skills relate to individual differences in children's mathematical skills? A review of evidence from brain and behavior. Trends in Neuroscience and Education, 2 (2), 48-55.

Desoete, A., Ceulemans, A., De Weerdt, F., \& Pieters, S. (2010). Can we predict mathematical learning disabilities from symbolic and non-symbolic comparison tasks in kindergarten? Findings from a longitudinal study. British Journal of Educational Psychology, 82, $64-81$.

Dillon, M. R., Kannan, H., Dean, J. T., Spelke, E. S. \& Duflo, E. (2017). Cognitive Science in the field: A preschool intervention durably enhances intuitive but not formal mathematics. Science, 357 (6346), 47-55.

Fazio, L.K., Bailey, D.H., Thompson, C.A., \& Siegler, R.S. (2014). Relations of different types of numerical magnitude representations to each other and to mathematics achievement. Journal of Experimental Child Psychology, 123, 53-72. 
Feigenson, L., Libertus, M.E., \& Halberda, J. (2013). Links between the intuitive sense of number and formal mathematics ability. Child Development Perspectives, 7 (2), 74 79.

Fuhs, M.W., \& McNeil, N. M. (2013). ANS acuity and mathematics ability in preschoolers from low-income homes: contributions of inhibitory control. Developmental Science, $16,136-148$.

Gilmore, C., Attridge, N., \& Inglis, M. (2011). Measuring the approximate number system. Quarterly Journal of Experimental Psychology, 64 (11), 2099-2109.

Gilmore, C., Attridge, N., Clayton, S., Cragg, L., Johnson, S. et al. (2013). Individual differences in inhibitory control, not non-verbal number acuity, correlate with mathematics achievement. PLOS ONE, 8 (6), e67374.

Gilmore, C., McCarthy, S.E., \& Spelke, E. (2007). Symbolic arithmetic without instruction. Nature, 447, 589-591.

Gordon, P. (2004). Numerical cognition without words: Evidence from Amazonia. Science, 306, 496-499.

Halberda, J \& Feigenson, L. (2008). Developmental change in the acuity of the 'number sense': The approximate number system in 3-, 4-, 5-, and 6-year-olds and adults. Developmental Psychology 44: 1457-1465.

Halberda, J., Ly, R., Wilmer, J.B., Naiman, D.Q., \& Germine, L. (2012). Number sense across the lifespan as revealed by a massive internet-based sample. Proceedings of the National Academy of Sciences, USA, 109 (28), 11116 - 11120.

Halberda, J., Mazzocco, M.M.M., \& Feigenson, L. (2008). Individual differences in nonverbal number acuity correlate with maths achievement. Nature, 455, 665-668.

Holloway, I.D. \& Ansari, D. (2009). Mapping numerical magnitudes onto symbols: The numerical distance effect and individual differences in children's math achievement. Journal of Experimental Child Psychology, 103, 17-29.

Hyde, D.C., Khanum, S., \& Spelke, E.S. (2014). Brief nonsymbolic, approximate number practice enhances subsequent exact symbolic arithmetic in children. Cognition, 131, 92- 107. Hyde, D.C., Spelke, E.S. (2011). Neural signatures of number processing in human infants: evidence for two core systems underlying numerical cognition: Neural signatures of number in infants. Developmental Science. 14(2):360-371.

Inglis, M., Attridge, N., Batchelor, S., \& Gilmore, C. (2011). Non-verbal number acuity correlates with symbolic mathematics achievement: but only in children. Psychonomic Bulletin \& Review, 18 (6), 1222-1229.

Izard V, Sann C, Spelke E.S., Streri, A. (2009). Newborn infants perceive abstract numbers. Proceedings of the National Academy of Sciences of the United States of America. 106(25):10382-10385.

Khanum, S. \& Hanif, R. (2014). Numerical acuity of American and Pakistani first-grade children: a cross-cultural comparison. Journal of Pakistan Psychiatric Society, 11 (2).

Khanum, S., Hanif, R., Berteletti, I., Spelke, E.S., \& Hyde, D.C. (2016). Effects of nonsymbolic approximate number practice on symbolic number abilities in Pakistani children. PLoS ONE, 11(10): e0164436.

Landerl, K., \& Kölle, C. (2009). Typical and atypical development of basic numerical skills in elementary school. Journal of Experimental Child Psychology, 103, 546 -565.

Libertus, M., \& Brannon, E. (2010). Stable individual differences in number discrimination in infancy. Developmental Science,13 (6),900-906. PMCID: PMC2966022.

Libertus, M.E., \& Brannon, E.M. (2009). Behavioral and neural basis of number sense in infancy. Current Directions in Psychological Science, 18 (6), 346 - 351. PMCID: PMC2857350.

Libertus, M.E., Feigenson, L., \& Halberda, J. (2011). Preschool acuity of the approximate number system correlates with school math ability. Developmental Science, 14 (6), $1292-1300$.

Libertus, M.E., Feigenson, L., \& Halberda, J. (2013a). Is approximate number precision a stable predictor of math ability? Learning and Individual Differences, 25, 126-133. 
Libertus, M.E., Feigenson, L., \& Halberda, J. (2013b). Numerical approximation abilities correlate with and predict informal but not formal mathematics abilities. Journal of Experimental Child Psychology, 116, 829-838.

Libertus, M.E., Odic, D., \& Halberda, J. (2012). Intuitive sense of number correlates with math scores on college entrance examination. Acta Psychologica, 141 (3), 373-379.

Lipton, J. S., \& Spelke, E. S. (2003). Origins of number sense: Large number discrimination in human infants. Psychological Science, 14, 396-401.

Lyons, I.M., Ansari, D., \& Beilock, S.L. (2012). Symbolic estrangement: evidence against a strong association between numerical symbols and the quantities they represent. Journal of Experimental Psychology: General, 141, 635-641.

Mazzocco, M.M.M., Feigenson, L., \& Halberda, J. (2011a). Impaired acuity of the approximate number system underlies mathematical learning disability (dyscalculia). Child Development, 82 (4), 1224-1237.

Mazzocco, M.M.M., Feigenson, L., \& Halberda, J. (2011b). Preschoolers' precision of the approximate number system predicts later school mathematics performance. PLoS ONE, 6 (9), e23749.

Mundy, E., Gilmore C. K. \& Spelke, E.S. (2009). Children's mapping between symbolic and nonsymbolic representations of number. J. Exp. Child Psychol. 103 490-502.

Nieder, A., Dehaene, S. (2009) Representation of number in the brain. Annu Rev Neu- rosci 32:185-208.

Odic, D., Libertus, M. E., Feigenson, L., \& Halberda, J. (2013). Developmental Change in the Acuity of Approximate Number and Area Representations. Developmental Psychology, 49(6), 1103-1112.

Park, J., \& Brannon, E.M. (2013). Training the approximate number system improves math proficiency. Psychological Science, 24 (10), 2013-2019.

Park, J., \& Brannon, E.M. (2014). Improving arithmetic performance with number sense training: an investigation of underlying mechanism. Cognition, 133, 188-200.

Piazza, M., Facoetti, A., Trussardi, A. N., Berteletti, I., Conte, S., Lucangeli, D., ...Zorzi, M. (2010). Developmental trajectory of number acuity reveals a severe impairment in developmental dyscalculia. Cognition, 116(1), 33-41.

Piazza, M., Pica, P., Izard, V., Spelke, E.S., \& Dehaene, S. (2013). Education enhances the acuity of the nonverbal approximate number system. Psychological Science, 24 (6), $1037-1043$.

Pica P., Lemer C., Izard W., Dehaene S. (2004). Exact and approximate arithmetic in an Amazonian indigene group. Science 306 499-503.

Rousselle, L. \& Noël, M-P. (2007). Basic numerical skills in children with mathematics learning disabilities: A comparison of symbolic versus non-symbolic number magnitude processing. Cognition, 102, 361-395.

Sasanguie, D., De Smedt, B., Defever, E., \& Reynvoet, B. (2012). Association between basic numerical abilities and mathematics achievement. British Journal of Developmental Psychology, 30, 344-357.

Sasanguie, D., Gobel, S.M., Moll, K., Smets, K., \& Reynvoet, B. (2013). Approximate number sense, symbolic number processing, or number-space mappings: what underlies mathematics achievement? Journal of Experimental Child Psychology, 114, 418-431.

Sasanguie, D., Van den Bussche, E., \& Reynvoet, B. (2012). Predictors for mathematics achievement? Evidence from a longitudinal study. Mind, Brain and Education, 6 (3), 119-128.

Schneider, M., Beeres, K., Coban, L., Merz, S., Susan Schmidt, S., Stricker, J., \& De Smedt, B. (2017). Associations of non-symbolic and symbolic numerical magnitude processing with mathematical competence: A meta-analysis. Developmental Science, 20(3), e12372.

Soltesz, F., Szücs, D., \& Szücs, L. (2010). Relationships among magnitude representation, counting, and memory in 4 - to 7- year - old children: a developmental study. Behavioral and Brain Functions, 6, 13. 
Starr, A., Libertus, M.E., \& Brannon, E.M. (2013). Number sense in infancy predicts mathematical abilities in childhood. Proceedings of the National Academy of Sciences, USA, 110 (45), 18116-18120.

Vanbinst, K., Ghesquiere, P., \& De Smedt, B. (2012). Numerical magnitude representations and individual differences in children's arithmetic strategy use. Mind, Brain and Education, 6 (3), 129-136.

Vanbinst, K., Ghesquiere, P., \& De Smedt, B. (2015). Does numerical processing uniquely predict first graders' future development of single-digit arithmetic? Learning and Individual Differences, 37, 153-160.

$\mathrm{Xu}, \mathrm{F}$., \& Spelke, E. S. (2000). Large number discrimination in 6-month-old infants. Cognition, 74, B1-B11. 